\title{
Echocardiographic Datasets Showing Development of Cardiac Remodelling in Rats at Different Time-points after Acute Myocardial Infarction
}

\section{Iordanis Mourouzis, Constantinos Pantos ( $\square)$}

Department of Pharmacology, University of Athens, 75 Mikras Asias Ave.,11527 Goudi, Athens, Greece

http://www.uoa.gr

\section{ARTICLE INFO: \\ ReCEIVED: 18 Oct 2015 \\ REVISED: 12 Nov 2015 \\ ACCEPTED: 30 Nov 2015 \\ ONLINE: 22 Dec 2015}

\section{KEYWORDS: \\ myocardial infarction \\ heart failure \\ cardiac remodelling \\ echocardiography \\ rat}

\begin{abstract}
The simulation of the time course of development of heart failure after an acute myocardial infarction requires large, open-access datasets. In this report, we present large echocardiographic datasets of cardiac function in rats after myocardial infarction at two different time points ( 2 and 13 weeks). We present measurements that show the deterioration of global left ventricular (LV) function, regional function, development of LV dilation, reactive hypertrophy and changes in LV geometry. We present in addition measurements showing the degree of cardiac injury assessed by the area and weight of the scar tissue. These data represent the progressive nature of cardiac remodelling and could be used to build computational models to predict the development of heart function deterioration based on the extent of myocardial injury and the development of LV remodelling.
\end{abstract}

(c) (7) (-)

(C) 2015 Hosting by Procon Ltd. All rights reserved.

\section{Overview}

The cardiac function is reduced after myocardial infarction due to myocardial injury and to changes in the viable non ischaemic myocardium, a process known as cardiac remodelling. This response is characterized by the development of cardiac hypertrophy, altered cardiac chamber geometry, shift of contractile proteins' expression to foetal pattern, switch to glucose metabolism and the induction of fibrosis. ${ }^{1,2}$

Current treatment of patients with acute myocardial infarction (AMI) reduces infarct size, preserves left ventricular function, and increases survival rates. However, it does not prevent remodelling which leads to heart failure. ${ }^{3,4}$ In fact, up to
$30-46 \%$ of the patients fail to improve functional recovery until six months after $\mathrm{AMI}$, despite the current anti-remodelling treatment. ${ }^{4,5}$ This prompts for further understanding of the pathophysiology of cardiac remodelling in order to predict disease progress and apply effective treatments tailored to the needs of the specific patient. $^{6}$

Modelling can be a powerful tool for analysing the complex interactions among changes in contractile proteins' expression, cardiac chamber geometry and wall tension caused by $\mathrm{AMI}$ and the therapeutic interventions modifying the existing dynamic balance that leads to progressive development of heart failure. The development of such 
holistic models taking into consideration changes at the level of cell, tissue and organ needs to be supplied with large-scale datasets from cell cultures, ex vivo and in vivo experimental models and clinical trials. ${ }^{7,8}$

In this paper, we present echocardiographic datasets from male Wistar rats that were subjected to acute myocardial infarction without reperfusion in order to study the development of cardiac remodelling. Animals were studied at two different time-points early ( 2 weeks) and late (13 weeks) after coronary artery ligation. Our group has worked for many years in this area to determine novel pathophysiological insights and therapeutic interventions using in vivo models of myocardial infarction in mice and rats. ${ }^{9-12}$

\section{Methods}

\section{Animals}

Male Wistar rats were maintained on a $12 \mathrm{~h}$ light/ dark cycle. Handling of animals was performed in accordance with the Guide for the Care and Use of Laboratory Animals published by the US National Institutes of Health Guide (NIH Pub. No. 83-23, Revised 1996). University ethics review board approved this experimental protocol.

\section{Experimental model of myocardial infarction in rats}

Surgical ligation of the left coronary artery was performed in order to generate myocardial infarction as described in the previous paper in this issue. Initial anaesthesia was achieved with injection of ketamine $(70 \mathrm{mg} / \mathrm{kg}$, i.p.) and midazolam $(0.1 \mathrm{mg} / \mathrm{kg}$, i.p.). Rats were subjected to intubation via a tracheal cannula and ventilated with a small rodent ventilator (Harvard Apparatus, Inspira, 50 breaths per min, $1 \mathrm{ml}$ per $100 \mathrm{~g}$ tidal volume). Inhaled sevoflurane at doses 1-2\% was used to maintain anaesthesia during surgery. A 60 silk suture was utilized for ligation of the left coronary artery. During the procedure, ECG recording was applied in order to monitor changes in heart rate and verify ST-segment changes typical of myocardial infarction. Body temperature was kept constant with a Harvard Homeothermic blanket. The animals were left to recover for 2 or 13 weeks after myocardial infarction. The same procedure was followed for sham-operated animals, but the coronary artery was not ligated. Animals were 12-16 weeks old at the time of surgical procedure. A total of 64 animals were subjected in sham-operation, while 74 animals were subjected in coronary artery ligation (CAL).

\section{Experimental procedure}

At the end of the experiment, animals were anaesthetized with ketamine hydrochloride and midazolame, subjected to echocardiography analysis and the heart was removed. Left ventricle (LV) of the heart was weighted and scar LV tissue was dissected out. The area of the scar tissue was measured in $\mathrm{mm}^{2}$, and the weight in $\mathrm{mg}$.

\section{Echocardiography}

Acquisition of echocardiographic images in short and long-axis was achieved with a Vivid 7 version Pro ultrasound system (GE Healthcare) equipped with a 14.0-MHz probe (i13L). Images were evaluated by two independent, experienced operators blinded to the experimental groups.

Estimated parameters include LV diameter at end-diastolic phase (LVIDd) and end-systolic phase (LVIDs), posterior wall thickness at end-diastolic phase (LVPW) and the ejection fraction (EF). The Simpson equation was used for calculation of EF. $\mathrm{EF} \%$ was used to determine the global contractile LV function. Systolic velocity of posterior wall radial displacement (SVPW) was determined and used to assess the regional contractile function of the LV myocardium. SVPW was measured from two-dimensional guided M-mode recordings obtained at the midventricular level. SVPW was calculated according to the following formula:

$$
\mathrm{V}=\mathrm{ds} / \mathrm{dt} \text {, }
$$

where $\mathrm{V}$ represents velocity, $\mathrm{s}$ - the distance, and $\mathrm{t}$ represents time.

\section{Data Records}

The data in the accompanying spreadsheet is organised in the following columns:

A: It shows the ID of the subject.

B: It shows the intervention that the animals were subjected to. It includes two options - either sham-operation or coronary artery ligation (CAL).

C: It shows the duration of the time in weeks between the intervention and evaluation of effects.

D: It shows body weight of animals in grams at the day of sacrifice.

E: It shows the left ventricular (LV) end-diastolic diameter in $\mathrm{cm}$ that was measured by echocardiography.

F: It shows the left ventricular end-systolic diameter in $\mathrm{cm}$ that was measured by echocardiography. 
G: It shows the left ventricular posterior wall thickness in $\mathrm{cm}$ that was measured by echocardiography.

$\mathrm{H}$ : It shows the maximum distance from the basis to the apex of the left ventricle in $\mathrm{cm}$ (called long axis) that was measured by echocardiography.

I: It shows the left ventricular ejection fraction in $\%$ that was measured by echocardiography using the Simpson equation.

$\mathrm{J}$ : It shows the systolic velocity of the left ventricular posterior wall in $\mathrm{cm} / \mathrm{sec}$ that was measured by echocardiography.

K: It shows the Wall tension index (WTI) defined as the ratio of (LV end-diastolic diameter)/ $2 *$ (left ventricular posterior wall thickness). WTI was measured in order to indirectly assess myocardial wall stress.

L: It shows the Sphericity Index (SI) defined as the ratio of long axis diameter of LV to LV end-diastolic diameter.

M: It shows the heart rate in beats per min that was measured during echocardiography.

$\mathrm{N}$ : It shows the left ventricular weight in grams.

O: It shows the weight of the scar tissue of the left ventricle in grams. Animals subjected to sham operation did not have a scar.

P: It shows the area of the scar tissue of the left ventricle in $\mathrm{mm}^{2}$. Animals subjected to sham operation did not have a scar.

\section{Use and Potential Reuse of the Dataset}

The dataset published in this paper was created from rats subjected to acute myocardial infarction (AMI) by ligation of the left coronary artery without reperfusion in order to study cardiac remodelling. Changes in LV morphology and function were evaluated by echocardiography early, at 2 weeks, and late - at 13 weeks. In this data paper, we present measurements that show the deterioration of global LV function (ejection fraction), regional function (systolic velocity of posterior wall), development of LV dilation (LV end-diastolic diameter and volume) and hypertrophy (LV weight and posterior wall thickness) as well as changes in LV geometry (sphericity index). At the same time, we include measurements that are considered to be important determinants of cardiac function such as the degree of cardiac injury (as assessed by the scar weight and area) and the heart rate.
Large, open access datasets, like this one, could serve as input for mathematical models that are capable to infer a function from observed data. ${ }^{13,14}$ This is particularly useful with several biological processes, where the complexity of the process makes the prediction of such a function very difficult with other methods. Thus, the field of machine learning and cognitive science is based on mathematical models that are used to estimate or approximate functions that depend on a large number of inputs (systems that learn from data). These systems are trained with real data to solve a wide variety of tasks that are hard to solve using ordinary rule-based programming. ${ }^{15}$ Such modelling approaches could be developed to enable the simulation of the pathophysiological process after an Acute Myocardial Infarction (AMI) that may lead to either early/late onset of heart failure (HF) or recovery of cardiac function. A modelling system medicine approach trained with such datasets could be expected to predict with accuracy the effects of novel or current treatments that act via modulation of tissue injury, LV dilation, LV geometry, hypertrophy, and regional contractile function. Some efforts have been made to simulate post-infarction remodelling processes. ${ }^{16,17}$

Recently a new model has been introduced capable of accurately predicting wall thinning and dilation of the left ventricle as a function of the reduction in blood supply in coronary vessels. ${ }^{18}$ However, the goal to create a calibrated computational model of the timeline of myocardial infarction able to predict the propensity towards heart failure has not been reached yet. The publication of open access datasets from controlled experimental studies is expected to be an assistant tool towards this goal. Furthermore, in silico simulation of experimental and clinical studies could be expected to facilitate streamlining for the drug development process and could enable prediction of novel drug effects to stratify patients prior to full scale clinical trials, potentially reducing costs and increasing the likelihood of a successful outcome.

\section{Conclusions}

In conclusion, we present echocardiographic datasets from male Wistar rats that were subjected to acute myocardial infarction without reperfusion at two different time-points - early (at 2 weeks) and late (at 13 weeks) after coronary artery ligation. Publication of such datasets could 
be the basis to create in silico disease models for heart failure after myocardial infarction.

\section{Data Files}

The data described in this paper is presented in an openly accessible spreadsheet: "01302_Rat AMI_Dataset.xIsx."

The file is available also in the Open Document Spreadsheet format.

\section{Acknowledgements}

The preparation of the manuscript of this data paper was supported by the EU-funded project OpenScienceLink (Grant agreement 318652).

\section{References}

1 Rajabi M, Kassiotis C, Razeghi P, Taegtmeyer H. Return to the fetal gene program protects the stressed heart: a strong hypothesis. Heart Fail Rev 2007;12:331343. doi: 10.1007/s10741-007-9034-1.

2 Swynghedauw B. Molecular mechanisms of myocardial remodeling. Physiol Rev 1999;79:215-262. PMID: 9922372.

3 Lymvaios I, Mourouzis I, Cokkinos DV, Dimopoulos MA, Toumanidis ST, Pantos C. Thyroid hormone and recovery of cardiac function in patients with acute myocardial infarction: a strong association? Eur J Endocrinol 2011;165:107-114. doi: 10.1530/EJE-110062.

${ }^{4}$ Springeling T, Kirschbaum SW, Rossi A, Baks T, Karamermer $\mathrm{Y}$, Schulz $\mathrm{C}$ et al. Late Cardiac Remodeling After Primary Percutaneous Coronary Intervention. Circ J 2012. doi: 10.1253/circj.CJ-12-0043.

5 Bolognese L, Neskovic AN, Parodi G, Cerisano G, Buonamici $P$, Santoro GM et al. Left ventricular remodeling after primary coronary angioplasty: patterns of left ventricular dilation and long-term prognostic implications. Circulation 2002;106:23512357. doi: 10.1161/01.CIR.0000036014.90197.FA.

6 Dargie H. Heart failure post-myocardial infarction: a review of the issues. Heart 2005 May; 91(Suppl 2): ii3-ii6. doi: 10.1136/hrt.2005.062018.

7 Amanfu RK, Saucerman JJ. Cardiac models in drug discovery and development: a review. Crit Rev Biomed Eng. 2011;39(5):379-95. doi: 10.1615/CritRevBiomed Eng.v39.i5.30.

8 Williams GS, Smith GD, Sobie EA, Jafri MS. Models of cardiac excitation-contraction coupling in ventricular myocytes. Math Biosci. 2010 Jul; 226(1):1-15. doi: 10.1016/j.mbs.2010.03.005.

9 Mourouzis I, Giagourta I, Galanopoulos G, Mantzouratou P, Kostakou E, Kokkinos AD, Tentolouris
$\mathrm{N}$,Pantos C. Thyroid hormone improves the mechanical performance of the post-infarcted diabetic myocardium: a response associated with up-regulation of $\mathrm{Akt} / \mathrm{mTOR}$ and AMPK activation. Metabolism. 2013 Oct;62(10):1387-93. doi: 10.1016/j.metabol. 2013.05.008.

${ }^{10}$ Pantos C, Mourouzis I. Translating thyroid hormone effects into clinical practice: the relevance of thyroid hormone receptor $\alpha 1$ in cardiac repair. Heart Fail Rev. 2015 May;20(3):273-82. doi: 10.1007/s10741-0149465-4.

11 Mourouzis I, Mantzouratou P, Galanopoulos G, Kostakou E, Dhalla AK, Belardinelli L, Pantos C. The beneficial effects of ranolazine on cardiac function after myocardial infarction are greater in diabetic than in nondiabetic rats. J Cardiovasc Pharmacol Ther. 2014 Sep;19(5):457-69. doi: 10.1177/1074248414524481.

12 Mourouzis I, Kostakou E, Galanopoulos G, Mantzouratou P, Pantos C. Inhibition of thyroid hormone receptor $\alpha 1$ impairs post-ischemic cardiac performance after myocardial infarction in mice. Mol Cell Biochem. 2013 Jul;379(1-2):97-105. doi: 10.1007/s11010-013-1631-9.

13 Isma'eel HA, Cremer PC, Khalaf S, Almedawar MM, Elhajj IH, Sakr GE, Jaber WA. Artificial neural network modeling enhances risk stratification and can reduce downstream testing for patients with suspected acute coronary syndromes, negative cardiac biomarkers, and normal ECGs. Int J Cardiovasc Imaging. 2015 Dec 1. doi: 10.1007/s10554-015-0821-9.

14 Tanaka H. Modeling the motor cortex: Optimality, recurrent neural networks, and spatial dynamics. Neurosci Res. 2015 Nov 10. pii: S0168-0102(15)002631. Review. doi: 10.1016/j.neures.2015.10.012.

15 Kourou K, Exarchos TP, Exarchos KP, Karamouzis MV, Fotiadis DI. Machine learning applications in cancer prognosis and prediction. Comput Struct Biotechnol J. 2014 Nov 15;13:8-17. doi: 10.1016/j.csbj.2014.11.005.

16 Göktepe S, Abilez OJ, Parker KK, Kuhl E. A multiscale model for eccentric and concentric cardiac growth through sarcomerogenesis. J Theor Biol. 2010;265(3):433-42. doi: 10.1016/j.jtbi.2010.04.023.

17 Lee LC, Wall ST, Genet M, Hinson A, Guccione JM. Bioinjection treatment: effects of post-injection residual stress on left ventricular wall stress. J Biomech. 2014 Sep 22;47(12):3115-9. doi: 10.1016/j.jbiomech.2014.06.026.

18 Sáez P, Kuhl E. Computational modeling of acute myocardial infarction. Comput Methods Biomech Biomed Engin. 2015 Nov 19:1-9. [Epub ahead of print] doi: 10.1080/10255842.2015.1105965. 